Proceedings of the 1996 IEEE

International Conference on Robotics and Automation

Minneapolis, Minnesota - April 1996

\title{
Holonomic and Omnidirectional Vehicle with Conventional Tires
}

\author{
Masayoshi Wada and Shunji Mori \\ Mechatronics Development Group, Electronics Development Laboratory \\ Fuji Electric Corporate Research and Development, Ltd. \\ 1, Fuji-machi, Hino-shi, Tokyo 191, JAPAN \\ E-mail : wada@fd013d03.tlab.fujidenki.co.jp
}

\begin{abstract}
A new type of holonomic omnidirectional vehicle which consists of wheel mechanisms without free rollers but with conventional tires is presented.

We first present a concept of the new driving wheel mechanism with a tire in the conventional style such as a rubber tire or a pneumatic tire,"Offset Steered Driving Wheel". The configuration of the wheel has an offset distance between $a$ wheel axle and a steering axle along a direction of wheel traveling. We then describe the kinematics and the control method of Offset Steered Driving Wheel to provide an omnidirectional and holonomic capability for a vehicle by actuating $a$ wheel axis and a steering axis independently. The wheel is non-holonomic system however, we introduce a control strategy to avoid a non-holonomic constraint. Simulation results of the single driving wheel and the twowheeled vehicle are performed to test the control method. Wheel design using conventional tire enables the mechanism to be simple, the motion of the vehicle to be smooth with high payload capability, and the detection of the position and the orientation of the vehicle to be precise.

The concept of Offset Steered Driving Wheel is implemented and tested on a prototype of the holonomic omnidirectional vehicle. Experimental results demonstrate high mobility of the vehicle prototype.
\end{abstract}

\section{Introduction}

Usual wheeled mobile robots such as car-like vehicles or independent driven two-wheeled vehicles are non-holonomic systems and constrained their mobility by non-holonomic constraints: they cannot move sideways. The non-holonomic constraint causes difficulties of trajectory planning, and moving sequence designs : it needs complicated maneuvering sequences to bring a vehicle to a side position, and unusual feedback control strategies of the vehicle.

In the past, four wheel steering vehicle[7] has been developed. The vehicle has steering wheels with conventional tires and can move in the omnidirection by orienting all wheels in the same direction, and moreover it can rotate. The vehicle has omnidirectional mobility however, it is non-holonomic system. Therefore to track trajectories which include discontinuity of curvature, the vehicle must stop at the points and reorient its wheels in the desired direction: The vehicle can only move in the direction of orientation of wheel traveling at each instant.

In contrast to these non-holonomic vehicles, holonomic vehicles have many potential advantages over these nonholonomic vehicles. Holonomic vehicles can move in any direction immediately with any orientation of the vehicle and motions of holonomic vehicle can be designed independently in each 3DOF coordinates $(x, y, \phi)$. This feature leads the system to be controlled or stabilized easily by usual feedback control techniques. These features greatly simplify a total system.

A variety of omnidirectional holonomic vehicles have been researched and developed. There are two concepts of the developments of holonomic omnidirectional vehicle into the previous researches.

The first one is to develop a driving wheel unit which has one directional active traveling ability and has free (passive) mobility to another directions. The Universal wheel with decoupling mechanism[2], the VUTON [3], Orthogonal wheel[4], and the Ball wheel mechanism [5] have wheel mechanisms which have only one directional active traveling ability. All of these wheels have free rollers on its main wheel or crawlers except Ball wheel mechanism [5]. Omnidirectional mobility can be achieved by an arrangement of three or more numbers of wheels on a platform. It is the most cases that a practical vehicle has four these wheel units on account of layout or mechanical stability of the vehicle.

The second one is to develop a driving wheel unit which has two directional active traveling ability. The Omnitrack [6] whose wheel unit has a lot of balls driven by two actuators accomplishes omnidirectional mobility without passive mechanisms.

These holonomic vehicles have problems under the practical applications listed below.

1) : Complicated mechanism $[2],[3],[4],[5],[6]$.

2) : Low payload capability because of a reduced contact area $[2],[4],[5]$.

3) : Limited accuracy of a motion and of an estimation of the position and the orientation of the vehicle by deadreckoning because of difficulties in detecting precise points of contact [2],[4],[5] in case of rotation.

4) : Difficulties in keeping four points contact between a vehicle and a floor under environments with floor irregularities, in cases of four wheeled vehicles using $[3],[4],[5]$ without suspensions.

5) : Inadequacy for dusty environments because of the mechanism of direct driving or supporting of wheel surfaces [5],|6].

6) : Inadequacy for environments with a step because of small curvature of the sideward wheels $[2],[3],[5]$. 
To overcome these problems, we propose a new type of wheel mechanism for holonomic vehicles which based on the latter concept; two directional active traveling. The wheel whose configuration has an offset distance between a wheel axle and a steering axle along a direction of wheel traveling has two actuators and can equip the tires in a conventional style such as rubber tires or pneumatic tires.

First, we will describe a concept of Offset Steered Driving Wheel, then kinematics and behaviors of the wheel and a vehicle using the wheels will be analyzed. A mobility will be evaluated in the experiments of the prototype vehicle.

\section{Offset Steered Driving Wheel}

Conventional wheels for holonomic vehicles mentioned in the previous section are all holonomic wheels which can be moved in omnidirection by themselves with their orientation constant. Accordingly no vehicles have steering mechanisms for rotating the driving wheels on a platform. It is sufficient for the holonomic vehicle but is not always necessary that their wheels have holonomic mobility.

Considering a plate controlled by two supporting sticks, translational movements of the plate are generated as the average velocities between the two supports. And rotational movements of the plate are generated by moments between two supports. Therefore, to provide omnidirectional velocity for each supporting stick is also sufficient for the holonomic mobility of a vehicle.

Because a vehicle frame is supported by means of steering shafts of wheels, our objective is to move a steering shaft in arbitrary direction continuously.

Fig. 1 shows a series of steering wheels, conventional, sideways offset steered, and forward offset steered.

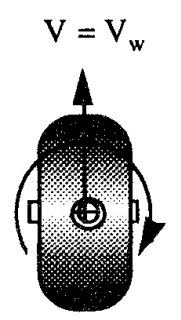

a) Conventional

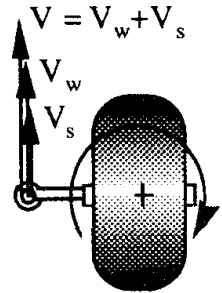

b) Offset Steered (Sideways)
Figure 1 : Series of Steered Driving Wheel

Conventional steered wheel (a) has two actuators for driving the wheel and the steering however, it can move only in one direction along the wheel traveling in the velocity $V_{w}$ at each instant. The reason is that the conventional steered wheel has a decoupling mechanical structure between the steering driving and a steering shaft motion: the steering shaft cannot be moved in the translational direction at all by a steering operation. The steering actuators are used only to rotate the wheels.

In order to couple the steering driving and the translational motion of a steering shaft, we introduce a remote center steering mechanism. Fig.1(b) illustrates a sideways offset steered wheel. In this case, the steering shaft can be moved around a point of contact in the velocity $\mathrm{Vs}$ according to a reaction of the steering torque. However, the steering shaft cannot be moved in an arbitrary direction because of a coincidence of the direction of each velocities generated by a steering motion and a wheel motion. In case of a forward offset steered wheel shown in Fig. 1(c), the direction of the velocity $V_{s}$ is perpendicular to the velocity $V_{w}$ at every moment. Therefore it is possible to translate a steering shaft in an arbitrary direction by controlling the wheel angular velocity $\mathrm{V}_{\mathrm{s}}$ and steering angular velocity $\mathrm{V}_{\mathrm{w}}$ independently by means of two actuators.

In following sections, the wheel shown in Fig.1(c) will be referred as "Offset Steered Driving Wheel". And we will analyze kinematics and the behavior of the wheel, then discuss about control method of the wheel and a two-wheeled vehicle.

\section{Kinematics and Control of Omnidirectional Vehicle}

\section{3-1 Single Driving Wheel}

To specify the configuration of the wheel, three generalized coordinates are needed. Kinematic equation of Offset Steered Driving Wheel shown in Fig. 2 can be obtained as relationships between coordinate vector $\mathbf{x}_{\mathbf{w}}$ and input vector $\mathbf{u}_{\mathrm{w}}$ as follows.

$$
\begin{aligned}
& {\left[\begin{array}{c}
\dot{x}_{w} \\
\dot{y}_{w} \\
\dot{\phi}_{w}
\end{array}\right]=\left[\begin{array}{cc}
r \cos \phi_{w} & -s \sin \phi_{w} \\
r \sin \phi_{w} & s \cos \phi_{w} \\
0 & 1
\end{array}\right]\left[\begin{array}{l}
\omega_{w} \\
\dot{\zeta}_{w}
\end{array}\right]} \\
& \rightarrow \dot{x}_{w}=\mathbf{B}_{w} \mathbf{u}_{w}
\end{aligned}
$$

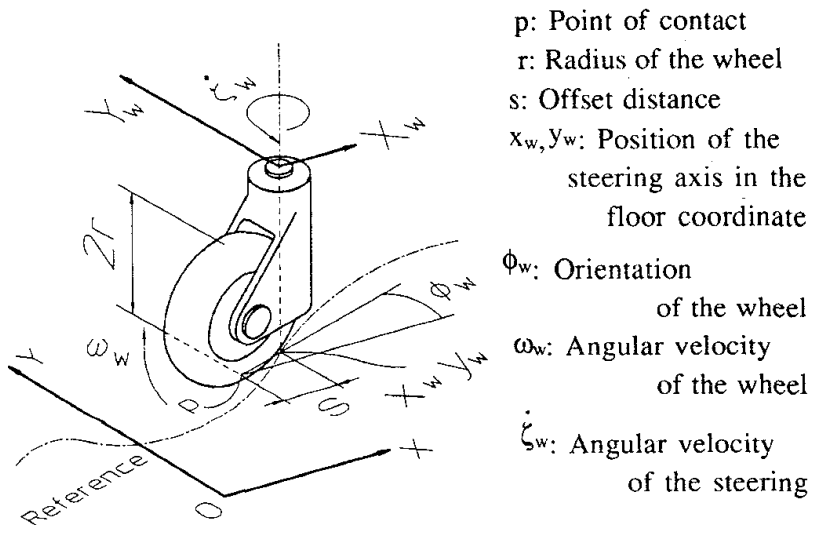

Figure 2 : Coordinate system of the single wheel

The wheel of this caster like type is known as nonholonomic system. The non-holonomic constraint of the system is derived by eq.(1) as 


$$
\begin{aligned}
& \sin \phi_{\mathrm{w}} \dot{\mathrm{x}}_{\mathrm{w}}-\cos \phi_{\mathrm{w}} \dot{\mathrm{y}}_{\mathrm{w}}+s \dot{\phi}_{\mathrm{w}}=0 \\
& \rightarrow \mathbf{G}_{\mathrm{w}} \dot{\mathbf{x}}_{\mathrm{w}}=0
\end{aligned}
$$

To calculate the input vector for the control of wheel coordinates, inverse kinematics of the system must be obtained. However $\mathbf{B}_{w}$ is not squire matrix, a least squire matrix can be obtained by using pseudo-inverse matrix.

$$
\begin{aligned}
\mathbf{u}_{\mathrm{w}} & =\mathbf{B}_{\mathrm{w}}^{-1} \dot{\mathbf{x}}_{\mathrm{w}} \\
=\left\{\left[\mathbf{B}_{\mathrm{w}}\right]^{\mathrm{T}} \mathbf{B}_{\mathrm{w}}\right\}^{-1}\left[\mathbf{B}_{\mathrm{w}}\right]^{\mathrm{T}} \dot{\mathbf{x}}_{\mathrm{w}} & =\left[\begin{array}{ccc}
\frac{1}{\mathrm{r}} \cos \phi_{\mathrm{w}} & \frac{1}{\mathrm{r}} \sin \phi_{\mathrm{w}} & 0 \\
-\frac{\mathrm{s}}{\mathrm{s}^{2}+1} \sin \phi_{\mathrm{w}} & \frac{\mathrm{s}}{\mathrm{s}^{2}+1} \cos \phi_{\mathrm{w}} & \frac{1}{\mathrm{~s}^{2}+1}
\end{array}\right]\left[\begin{array}{c}
\dot{\mathrm{x}}_{\mathrm{w}} \\
\dot{\mathrm{y}}_{\mathrm{w}} \\
\dot{\phi}_{\mathrm{w}}
\end{array}\right]
\end{aligned}
$$

Because this system is an overdetermined system, it is a quite difficult problem to obtain a control input vector $\mathbf{u}_{w}$ by using inverse kinematics eq.(3) so as to stabilize the wheel around a reference position $\left(x_{w}, y_{w}, \phi_{w}\right)$, which chosen in arbitrary, by mean of usual linear feedback control techniques[8]. This is a characteristics of the non-holonomic systems.

To avoid the problem of the non-holonomic system, we introduce a control strategy which reduce the numbers of controlled coordinates of the system according to the number of non-holonomic constraints. This system includes one constraint condition, therefore we can reduce the numbers of controlled coordinates from three to two.

Because our objective of the control of Offset Steered Driving Wheel is to move the steering shaft $\left(x_{w}, y_{w}\right)$ simultaneously in omnidirection, we give up the control of the coordinate $\dot{\phi}_{w}$; the angular velocity of wheel orientation. Now the kinematics of reduced coordinate system can be reconstructed from eq.(1) as follows

$$
\begin{aligned}
& {\left[\begin{array}{l}
\dot{x}_{w} \\
\dot{y}_{w}
\end{array}\right]=\left[\begin{array}{cc}
r \cos \phi_{w} & -s \sin \phi_{w} \\
r \sin \phi_{w} & s \cos \phi_{w}
\end{array}\right]\left[\begin{array}{l}
\omega_{w} \\
\zeta_{w}
\end{array}\right]} \\
& \rightarrow \dot{x}_{w 0}=\mathbf{B} \mathbf{w}_{w 0} \mathbf{u}_{w} \\
& \dot{\phi}_{w}=-\frac{1}{s} \sin \phi_{w} \dot{x}_{w}+\frac{1}{s} \cos \phi_{w} \dot{y}_{w}
\end{aligned}
$$

The coordinate $\dot{\phi}_{w}$ is treated as an internal variable which determined inevitably by the controlled coordinates $\dot{x}_{w}$ and $\dot{y}_{w}$ so as to satisfy the non-holonomic constraint $(4-2)$, the rewritten style of the eq.(2).

We can get only one control input vector $\left[\omega_{w}, \dot{\zeta}_{w}\right]^{\mathrm{T}}$ as a solution of inverse kinematics of the equation(4-1) with no singularity which generate the translational velocity of the steering shaft as

$$
\begin{aligned}
\mathbf{u}_{w} & =\mathbf{B}_{w 0}^{-1} \dot{\mathbf{x}}_{w 0} \\
& =\left[\begin{array}{cc}
\frac{1}{\mathbf{r}} \cos \phi_{w} & \frac{1}{\mathbf{r}} \sin \phi_{w} \\
-\frac{1}{\mathbf{s}} \sin \phi_{w} & \frac{1}{\mathbf{s}} \cos \phi_{w}
\end{array}\right]\left[\begin{array}{l}
\dot{x}_{w} \\
\dot{y}_{w}
\end{array}\right]
\end{aligned}
$$

By using proposed wheel mechanism and control method, the wheel can achieve a reproduction of "caster motion" by active driving. In Fig. 3 wheel simulation is shown whose steering shaft is moving along a straight line in the direction of behind 45 degrees. The wheel is moving backward at the beginning of the motion, then turning its orientation automatically, after that the rolling direction of the wheel is reversed and going forward along the line.

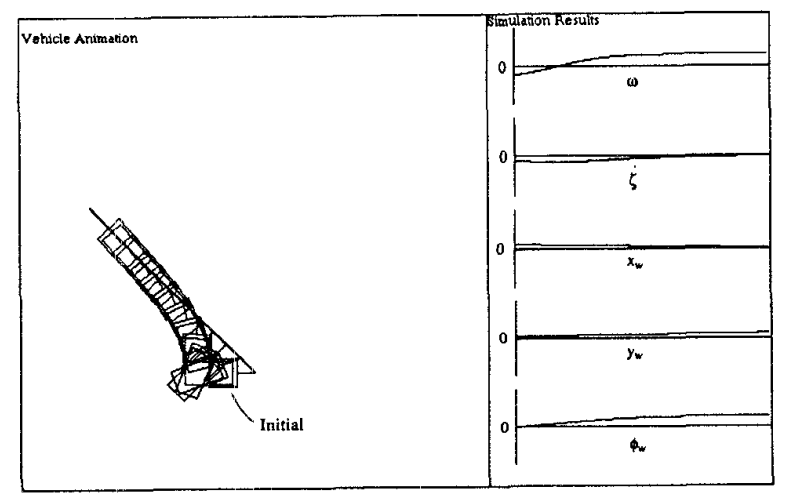

Figure 3 : Single Wheel Simulation

\section{3-2 Two Wheeled Vehicle}

In the previous section, it was mentioned that the proposed driving wheel can generate the velocity of the steering shaft in any directions on the two-dimensional field. A vehicle has 3DOF; 2DOF for translational and $1 D O F$ for rotational. Therefore it is necessary for the vehicle to have two or more numbers of proposed driving wheel. Fig. 4 shows a schematic of a two-wheeled vehicle which has a couple of Offset Steered Driving Wheels $A$ and $B$ with a distance $W$. The origin of the vehicle coordinate frame $\left(O-X_{v} Y_{v}\right)$ is at the midpoint between two steering axis of the wheels. We can input the omnidirectional and simultaneous motions of steering shaft of each wheel $A\left(\dot{x}_{a}, \dot{y}_{a}\right)$ and $B\left(\dot{x}_{b}, \dot{y}_{b}\right)$ to the vehicle. Then the kinematic model of the two wheeled vehicle can be obtained as follows.

$$
\begin{aligned}
& {\left[\begin{array}{c}
\dot{x}_{\mathrm{v}} \\
\dot{y}_{\mathrm{v}} \\
\dot{\phi}_{\mathrm{v}}
\end{array}\right]=\left[\begin{array}{cccc}
\frac{1}{2} & 0 & \frac{1}{2} & 0 \\
0 & \frac{1}{2} & 0 & \frac{1}{2} \\
\frac{1}{\mathrm{~W}} \cos \phi_{\mathrm{v}} & \frac{1}{\mathrm{~W}} \sin \phi_{\mathrm{v}} & -\frac{1}{\mathrm{~W}} \cos \phi_{\mathrm{v}} & -\frac{1}{\mathrm{~W}} \sin \phi_{\mathrm{v}}
\end{array}\right]\left[\begin{array}{c}
\dot{x}_{\mathrm{a}} \\
\dot{\mathrm{y}}_{\mathrm{a}} \\
\dot{x}_{\mathrm{b}} \\
\dot{y}_{\mathrm{b}}
\end{array}\right]} \\
& \rightarrow \dot{\mathbf{x}}_{\mathrm{v}}=\mathbf{B}_{\mathrm{v}} \mathbf{u}_{\mathrm{v}}
\end{aligned}
$$

This system includes four inputs and three outputs however, there exist a geometric constraint condition between $\left(\dot{x}_{a}, \dot{y}_{a}\right)$ and $\left(\dot{x}_{b}, \dot{y}_{b}\right)$ : it keeps a constant distance $W$. Therefore the system is a determined system. The input vector $u_{v}$ can easily obtained from the vector $\dot{x}_{v}$ as

$$
\begin{aligned}
& \mathbf{u}_{\mathrm{v}}=\mathbf{R}_{\mathrm{v}} \dot{\mathbf{x}}_{\mathrm{v}} \\
& =\left[\begin{array}{cccc}
1 & 0 & \frac{\mathrm{W}}{2} \cos \phi_{v} \\
& 0 & 1 & \frac{\mathrm{W}}{2} \sin \phi_{v} \\
1 & 0 & -\frac{\mathrm{W}}{2} \cos \phi_{v} \\
0 & 1 & -\frac{\mathrm{W}}{2} \sin \phi_{v}
\end{array}\right]\left[\begin{array}{c}
\dot{\mathbf{x}}_{\mathrm{v}} \\
\dot{\mathrm{y}}_{\mathrm{v}} \\
\dot{\phi}_{\mathrm{v}}
\end{array}\right]
\end{aligned}
$$


Fig. 5 shows a simulation result of the two-wheeled vehicle executing a constant translational motion with a constant rotational motion.

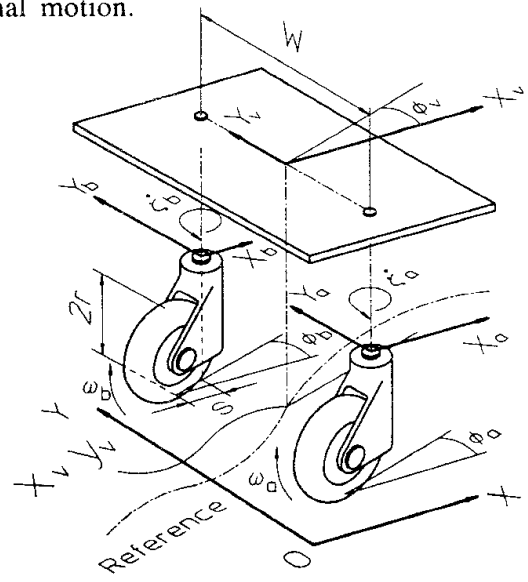

Figure 4 : Coordinate system of two-wheeled vehicle

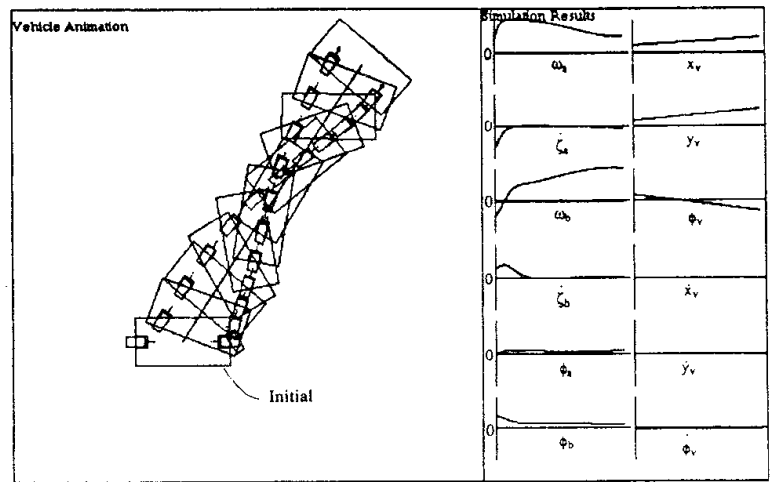

Figure 5 : Two-wheeled Vehicle Simulation

\section{Development of a prototype vehicle}

\section{4-1 Wheel mechanism}

A first prototype vehicle which based on the proposed methodology has been developed.

Fig.6 shows an overview of the wheel. To keep away from a problem of cables which across the continuously rotatable steering, a wheel actuator and a steering actuator are set up on the vehicle chassis and the driving torque is transmitted by a gear train.

Fig.7 illustrates the wheel driving mechanism of the prototype. Interferences of motions between the wheel axis and the steering axis occurs because of the setting of the wheel actuator on the chassis, but is compensated by an actuator control.

Fig. 8 shows an overview of the prototype vehicle. The vehicle has a couple of Offset Steered Driving Wheels on a diagonal position and a couple of caster wheels on the other diagonal position to carry the frame on the level.

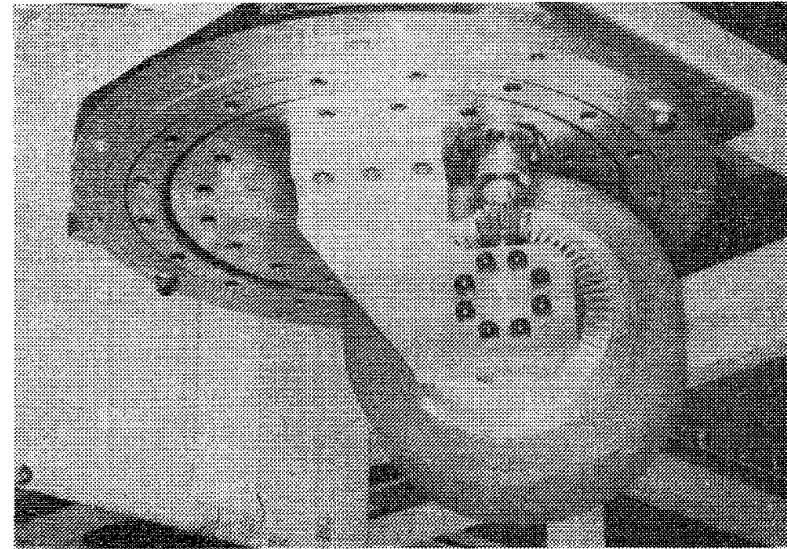

Figure 6: Offset Steered Driving Wheel Prototype

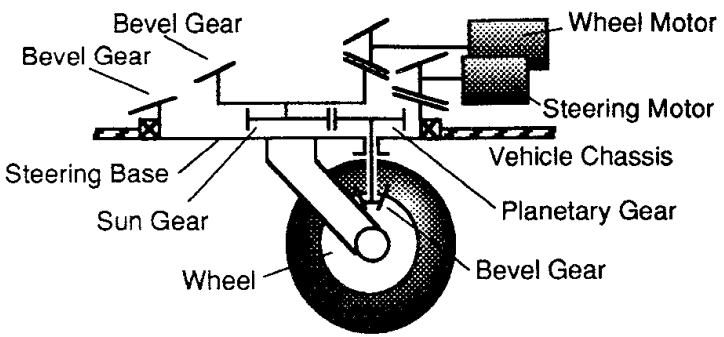

Figure 7: Wheel Driving Mechanism

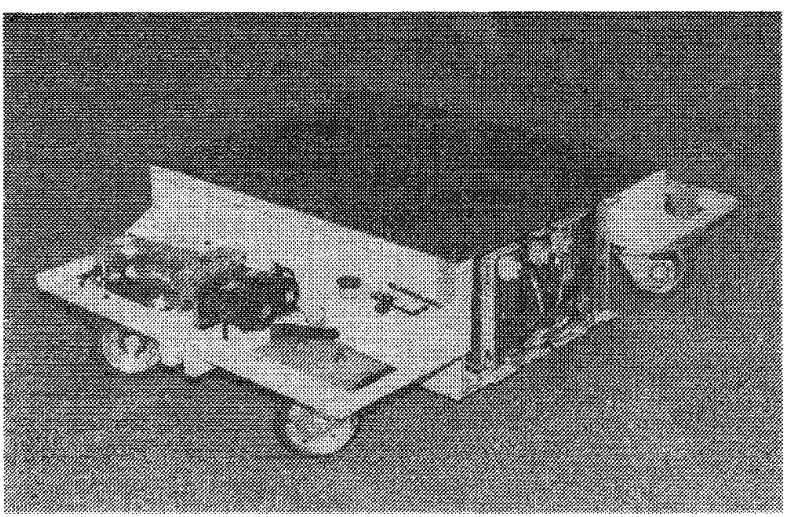

Figure 8: Omnidirectional Vehicle Prototype

Specifications of the prototype vehicle is as follows.

Vehicle Size: $750 \mathrm{~mm} \times{ }^{\mathrm{d}} 1150 \mathrm{~mm} \times{ }^{\mathrm{b}} 340 \mathrm{~mm}$ Weight: Approx. $115 \mathrm{~kg}$ Load capability : $300 \mathrm{~kg}$

Wheel Diameter: $\quad 150 \mathrm{~mm}$ Width : $\quad 60 \mathrm{~mm}$ Offset distance : $55 \mathrm{~mm}$ Actuator : $\quad$ AC servo motor $(300 \mathrm{~W})$

\section{4-2 Control system}

The control system of the vehicle, shown in Fig.9, has hierarchical structure consists of a vehicle level controller 
and wheel level controllers.

The Vehicle level controller receives vehicle velocity reference from main control system (a trajectory planner or a manual controller such as a 3 DOF joystick.) and calculates wheel unit references from vehicle references by using vehicle inverse kinematics eq.(7). Wheel unit level controllers receive wheel unit references from vehicle level controller and then calculate reference angular velocities of the wheel and the steering by using wheel inverse kinematics eq.(5). After the compensation of the mechanical interferences between wheel axis and steering axis, these angular velocity references of each axis are input to motor drivers used as actuators of the wheel.

The system is implemented by three board computers contain $80186 \mathrm{CPU}$ without co-processor connected by MultiBus II. One board (a main board) is used for a vehicle level controller, Another boards (slave boards) are used for wheel level controllers.

Vehicle motions are controlled by the system within 1 msec sampling time.

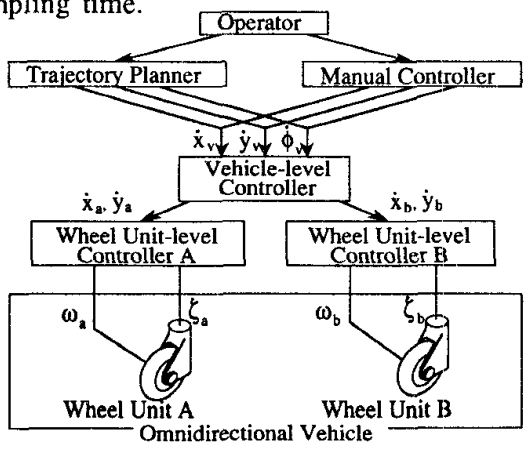

Figure 9 : Vehicle Control System

\section{Experiments of the Vehicle Prototype}

Mobility and a performance accuracy of the prototype vehicle was tested by a series of experiments

\section{5-1 Omnidirectional Mobility}

To demonstrate an omnidirectional mobility of the prototype vehicle, some patterns of example motions were performed. Experimental results are shown in Fig.10, 11, and 12. Small lights were mounted at the corners of a top plate of the vehicle diagonally. Actual paths of the vehicle are depicted by these lights.

Fig. 10 shows a crank motion with the orientation of the vehicle constant, going straight $1.25 \mathrm{~m}$, then moving sideways $1 \mathrm{~m}$, and going straight $1.25 \mathrm{~m}$ again. Fig. 11 shows a 360 degrees spin turn motion. Fig. 12 shows a $2.5 \mathrm{~m}$ translational motion with 1080 degrees ( 3 rounds) rotation of constant angular velocity.

\section{5-2 Holonomic Mobility and its Accuracy}

Fig.13 shows a schematic drawing of an experimental setup. In this experiment, the vehicle was programmed to move along a $1 \mathrm{~m}$ by $1 \mathrm{~m}$ triangular trajectory surrounded by an L-shaped corner. In order to measure the actual path, two ultrasonic distance sensors $(X, Y)$ were mounted at the corner of the vehicle frame perpendicular to each other. These sensors detected the distances to the walls along the each direction.

The vehicle traveled through the triangle path with its orientation constant in order $\mathrm{A}-\mathrm{B}-\mathrm{C}-\mathrm{A}$. The maximum speed of each run was approximately $0.13 \mathrm{~m} / \mathrm{sec}$ with constant acceleration and deceleration. Interval times was not set up at each turning points. Tracking control using dead-reckoning or any position detection system was not implemented in the experiments

Fig. 14 shows an experimental result of sensed distance in each direction. Simultaneous constant outputs of both $X$ and $Y$ sensors means that the vehicle stayed at the point. There was no point of the case except the point $A$. The direction of the vehicle motion was changed instantaneously at the points $\mathrm{B}$ and $\mathrm{C}$ without waiting a steering operation. This mobility is the characteristics of the holonomic vehicle.

We plotted the actual path shown in Fig.15. The worst position error, displacement between a start position and an end position, in ten trials was $64 \mathrm{~mm}$. It was $1.8 \%$ of the total trajectory distance, $(1+1+\sqrt{2}) \mathrm{m}$.

\section{Conclusion}

We have proposed Offset Steered Driving Wheel for an omnidirectional and holonomic vehicle. The wheel can be equipped not with the special tires but with the conventional tires such as rubber tires or pneumatic tires. The proposed vehicle is adequate for the practical applications because of the features mentioned as follows.

1): It has no special tires or special complex mechanisms.

2): High payload capability

3): Smooth motion

The kinematics of the wheel and the vehicle has been analyzed. And control method of the wheel has been proposed to avoid the non-holonomic problem of the wheel. Then simulations of single wheel and the vehicle was performed to test the control method. Using proposed control method, the wheel can achieve "caster motion" by means of active driving.

A holonomic omnidirectional vehicle prototype has been developed and designed. Smooth motions and the good performances of the prototype vehicle are demonstrated by the experiments.

\section{References}

[1] M.Wada, Y.Tominaga and S.mori,"Omnidirectional Holonomic Mobile Robot Using Nonholonomic Wheels," 1995 IEEE Int.Conf.on Intelligent Robots and Systems (IROS'95), pp446-453, Aug.1995.

[2] H.Asama, M.Sato, L.Bogoni, et al,"Development of an Omni-Directional Mobile Robot with 3 DOF Decoupling Mechanism," 1995 IEEE Int. Conf. on Robotics and Automation, pp1925-1930, May. 1995. 
[3] S.Hirose and S.Amano,"The VUTON: High Payload High Efficiency Holonomic Omni-Directional Vehicle", 6th Int. Symp. on Robotics Research, October 1993.

[4] F.G.Pin and S.M.Killough,"A New Family of Omnidirectional and Holonomic Wheeled Platforms for Mobile Robots," IEEE Transactions on Robotics and Automation, Vol.10, No.4, pp480-489, 1994.

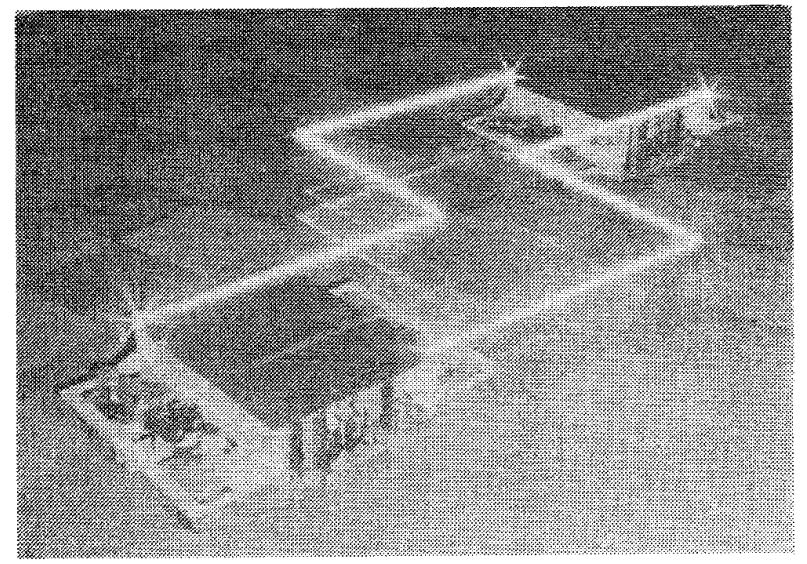

Figure 10: Crank Motion

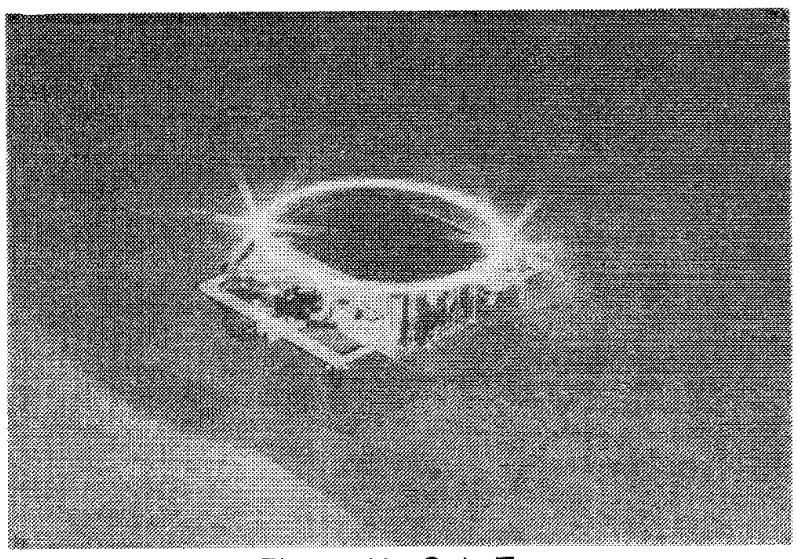

Figure $11:$ Spin Turn

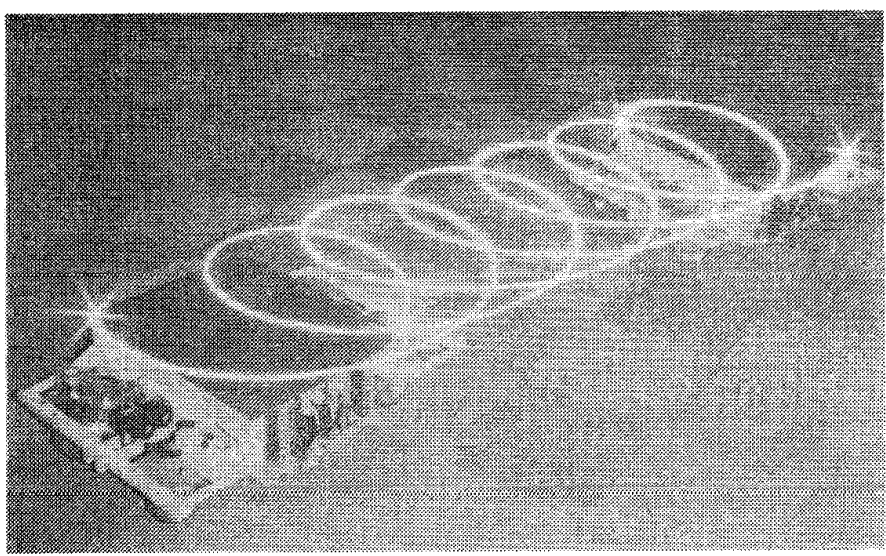

Figure 12 : Translational Motion with Rotation
[5] M.West and H.Asada,"Design and Control of Ball Wheel Omnidirectional Vehicles," 1995 IEEE Int. Conf. on Robotics and Automation, pp1931-1938, May.1995.[6] M.West and H.Asada,"Design of a Holonomic Omnidirectional Vehicle," 1992 IEEE Int. Conf. on Robotics and Automation, pp97-103, May. 1992.

[7] E. Nakano and N.Koyachi,"An Advanced Mechanism of the Omni-Directional Vehicle (ODV) and Its Application to the Working Wheelchair for the Disabled," 1983Int. Conf. on Advanced Robotics, pp277-284, 1983.

[8] Y.Zhao and S.L.BeMent,"Kinematics, Dynamics and Control of Wheeled Mobile Robots," 1992 IEEE Int. Conf. on Robotics and Automation, pp91-96, May. 1992.

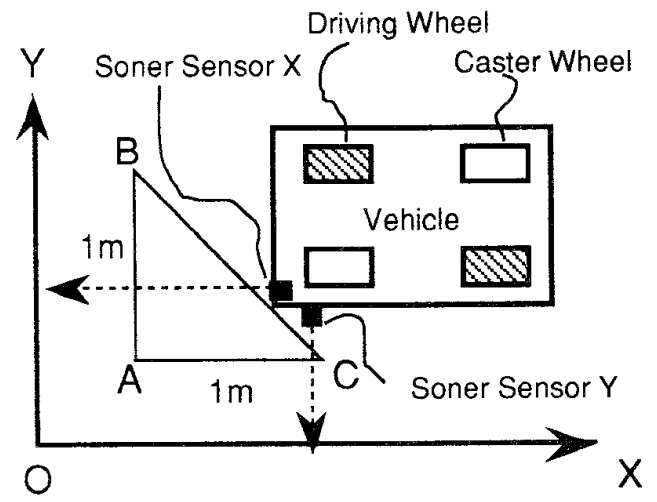

Figure 13 : Schematic Drawing of the Experiment

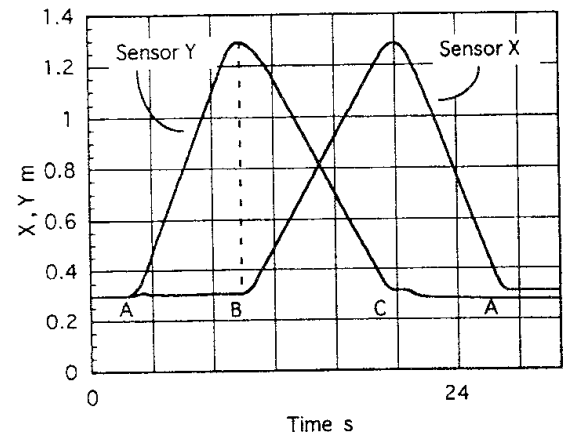

Figure 14 : Experimental Result (Sensor Output)

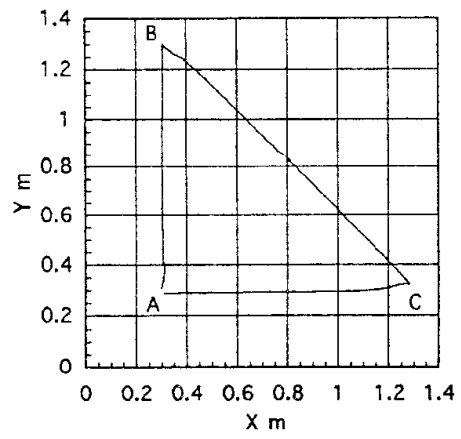

Figure 15 : Experimental Result (Vehicle Path) 